\title{
EEG-Based Subject- and Session-independent Drowsiness Detection: An Unsupervised Approach
}

\author{
Nikhil R. Pal, 1, 2, 3 Chien-Yao Chuang,, 2 Li-Wei Ko, 1, 2 Chih-Feng Chao, 1, 2 \\ Tzyy-Ping Jung, ${ }^{1,2,4}$ Sheng-Fu Liang, ${ }^{5}$ and Chin-Teng Lin ${ }^{1,2}$ \\ ${ }^{1}$ Department of Computer Science, National Chiao-Tung University, 1001 University Road, Hsinchu 30010, Taiwan \\ ${ }^{2}$ Brain Research Center, National Chiao-Tung University, 1001 University Road, Hsinchu 30010, Taiwan \\ ${ }^{3}$ Computer and Communication Sciences Division, Electronics and Communication Sciences Unit, Indian Statistical Institute, \\ 203 Barrackpore Trunk Road, Kolkata 700108, India \\ ${ }^{4}$ Institute for Neural Computation, University of California of San Diego, 4150 Regents Park Row, La Jolla, CA 92037, USA \\ ${ }^{5}$ Department of Computer Science and Information Engineering, National Cheng-Kung University, University Road, \\ Tainan 701, Taiwan
}

Correspondence should be addressed to Chin-Teng Lin, ctlin@mail.nctu.edu.tw

Received 2 December 2007; Revised 25 June 2008; Accepted 22 July 2008

Recommended by Chien-Cheng Lee

\begin{abstract}
Monitoring and prediction of changes in the human cognitive states, such as alertness and drowsiness, using physiological signals are very important for driver's safety. Typically, physiological studies on real-time detection of drowsiness usually use the same model for all subjects. However, the relatively large individual variability in EEG dynamics relating to loss of alertness implies that for many subjects, group statistics may not be useful to accurately predict changes in cognitive states. Researchers have attempted to build subject-dependent models based on his/her pilot data to account for individual variability. Such approaches cannot account for the cross-session variability in EEG dynamics, which may cause problems due to various reasons including electrode displacements, environmental noises, and skin-electrode impedance. Hence, we propose an unsupervised subject- and session-independent approach for detection departure from alertness in this study. Experimental results showed that the EEG power in the alpha-band (as well as in the theta-band) is highly correlated with changes in the subject's cognitive state with respect to drowsiness as reflected through his driving performance. This approach being an unsupervised and session-independent one could be used to develop a useful system for noninvasive monitoring of the cognitive state of human operators in attention-critical settings.
\end{abstract}

Copyright (c) 2008 Nikhil R. Pal et al. This is an open access article distributed under the Creative Commons Attribution License, which permits unrestricted use, distribution, and reproduction in any medium, provided the original work is properly cited.

\section{INTRODUCTION}

Drivers' fatigue is one of the primary causal factors for many road accidents and hence detection of drowsiness of drivers in real time can help preventing many accidents behind the steering wheel. In the field of safety driving, thus development of methodologies for detection drowsiness/departure from alertness in drivers has become an important area of research. Drowsiness leads to a decline in drivers' abilities of perception, recognition, and vehicle control, and hence monitoring of drowsiness in drivers is very important to avoid road accidents. It is known that various physiological factors covary with drowsiness levels [1-5]. Some such factors are eye activities, heart rate variability (HRV), and the electroencephalogram (EEG) activities. Since the effect of changes in cognitive state on EEG is quite strong, in this study we will use EEG as our information source for detection of drowsiness. Most of the earlier studies using EEG relating to assessment of changes in cognitive states are supervised in nature and have used the same detection model for all subjects [6-8]. But it is known that there existed relatively large subjective variability in EEG dynamics relating to drowsiness/departure from alertness. This suggests that for many operators, group statistics or a global model may not be effective to accurately predict changes in the cognitive states [9-12]. Subject-dependent models have also been developed to account for individual variability. Such personalised models although can alleviate 
the problem of individual variability in EEG spectra; such methods cannot take into account the variability between sessions in EEG spectra due to various factors such as electrode displacements, environmental noises, skin-electrode impedance, and baseline EEG differences. One of the major problems in dealing with EEG signals in a real-time driving environment is the presence of noise. Often independent component analysis (ICA) [13-17] is used for cleaning noise from EEG. However, selection of the noisy components in an automatic manner using ICA is still a difficult task.

In this investigation we introduce an unsupervised approach to estimate a model for the alert state of the subject. We will refer to such models as alert-models. A part of this investigation has been reported in [18]. The proposed approach can account for the variability in EEG signals between individuals and between sessions with the same individual. Being an unsupervised approach we do not need a teacher or a labeled training dataset with information on whether the driver is in an alert state or drowsy state at every time instant. In this approach, we derive models of the alert state of the subject as characterised by the EEG signal collected during the first few minutes of recording. We assume that during the first few minutes of driving, the driver (subject) will be in an alert state, although he/she may not be in a completely normal state as he/she might have walked some distance to reach the garage. This approach can account for baseline shifts and the variations in EEG spectra due to changes in recording conditions in different driving sessions. We find that the EEG log power in the alpha-band (as well as in the theta-band) and the driving performance exhibits a rough linear relation suggesting that changes in the cognitive state are reflected in the EEG power in the two specified bands. We then demonstrate that deviation of the EEG power from that of the alert model also follows a similar relation with the changes in driving performance, and hence with the changes in cognitive state. Consequently, a derivation from the alert model can be used to detect drowsiness and that is what we do in this investigation.

\section{DATA ACQUISITION}

\subsection{Experimental set up: a virtual reality (VR-)based driving environment}

In this study we use a virtual-reality-based highway-driving environment to generate the required data. Some of our previous studies to investigate changes in drivers' cognitive states during a long-term monotonous driving have also used the same VR-based environment [19, 20]. In this system, a real car mounted on a 6-degree-of-freedom Stewart platform is used for the driving and seven projectors are used to generate 3D surrounded scenes. During the driving experiments, all scenes move depending on the displacement of the car and the subject's maneuvering of the wheel, which makes the subject feel like driving the car on a real road. In all our experiments, we have kept the driving speed fixed at $100 \mathrm{~km} / \mathrm{h}$ and the system automatically and randomly drifts the car away from the center of the cruising lane to mimic the effects of a nonideal road surface. The driver is asked to

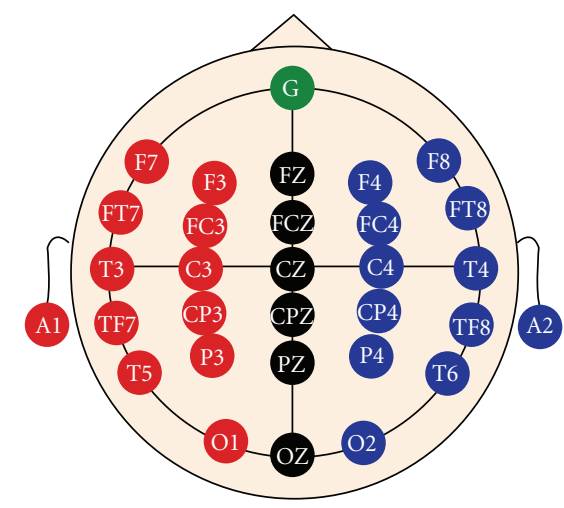

Figure 1: Electrodes placement of international 10-20 system. F: frontal lobe, T: temporal lobe, C: central lobe, P: parietal lobe, and O: occipital lobe. $\mathrm{Z}$ refers to an electrode placed on the midline.

maintain the car along the center of the cruising lane. All subjects involved in this study have good driving skill and hence when the subject is alert, his/her response time to the random drift is short and the deviation of the car from the center of the lane is small. But when the subject is not alert or drowsy, both the response time and the car's deviation are high. Note that, in all our experiments, the subject's car is the only car cruising on the VR-based freeway. Although both response time and the deviation from the central line are related to the subject's driving performance, in this study, we use the car's deviation from the central line as a measure of behaviour performance of the subjects.

\subsection{The EEG recording system}

The data acquisition system uses 32 sintered $\mathrm{Ag} / \mathrm{AgCl}$ EEG/EOG electrodes with a unipolar reference at right earlobe and 2 ECG channels in bipolar connection which are placed on the chest. All EEG/EOG electrodes were placed following a modified international 10-20 system and refer to right earlobe as depicted in Figure 1. In Figure 1, A1 and A2 are two reference channels. The two channels FP1 and FP2 are found to be quite noisy and hence we do not use the signals obtained from them. Thus, we use data from 28 channels. Before the data acquisition, the contact impedance between EEG electrodes and cortex was calibrated to be less than $5 \mathrm{k} \Omega$. We use the Scan NuAmps Express system (Compumedics Ltd., VIC, Australia) to simultaneously record the EEG/EOG data and the deviation between the center of the vehicle and the center of the cruising lane. The EEG data are recorded with 16-bit quantization level at the sampling rate of $500 \mathrm{~Hz}$. To reduce the burden of computation, the data are then downsampled to sampling rate of $250 \mathrm{~Hz}$. Since the objective is to develop methodologies that can be used in real time, we do not use sophisticated noise cleaning techniques such as ICA but we preprocess the EEG signals using a simple lowpass filter with a cutoff frequency of $50 \mathrm{~Hz}$ to remove the line noise $(60 \mathrm{~Hz}$ and its harmonics) and other highfrequency noise. 


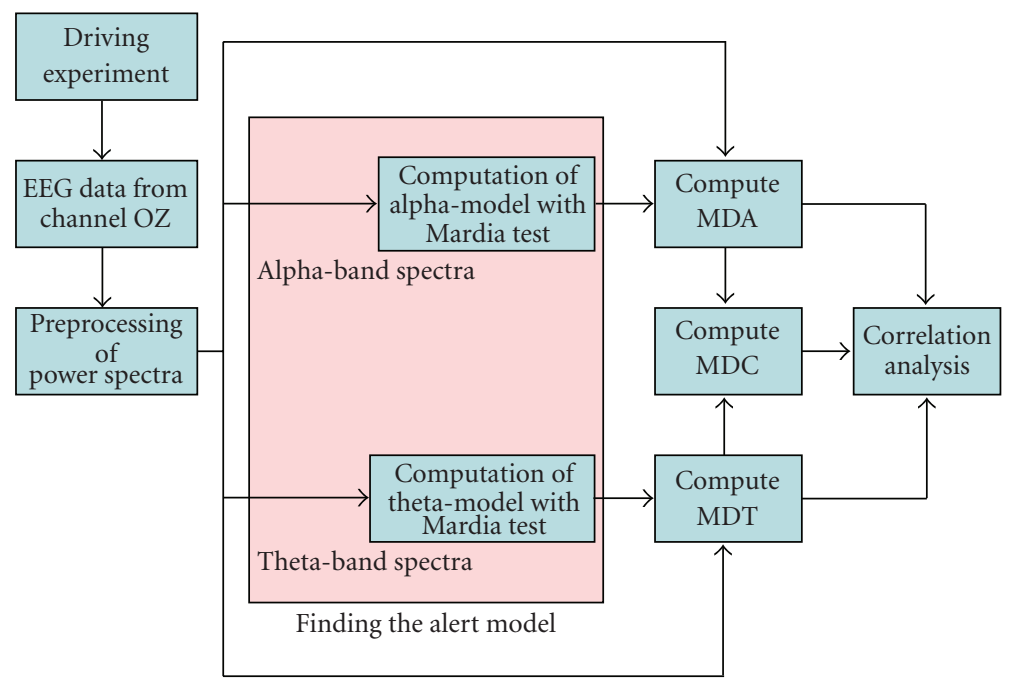

FIGURE 2: The flowchart of the EEG analysis method. First, we calculate power spectra of EEG data and preprocess with median filter. Then, we select theta- and alpha-band powers while the subject is alert to build two alert models. After the models are built, alpha- and theta-band powers are used to deviations $\left(\mathrm{MD}^{*}\right)$ from the models. We smooth the resultant $\mathrm{MD}^{*}$ with a 90 -second moving window at 2-second steps and calculate the correlations between subject's driving performance and the smoothed $\mathrm{MD}^{*}$.

\subsection{The subjects}

Here we provide a brief description of the EEG recording system as well as of the subjects involved in this study. We have used a set of thirteen subjects (ages varying from 20 to 40 years old) to generate data for the investigation. Of this thirteen, ten subjects are the same as used in [18]. Statistical reports [21] suggest that people often get drowsy within one hour of continuous driving in the early afternoon hours. Moreover, after a good sleep in the night, people are not likely to fall sleep easily during the first half of the day. And hence, we have conducted all our experiments in the early afternoon after lunch so that we can generate more useful data. We have informed the participants about the goal of these experiments and the general features of the driving task. We have also completed the necessary formalities to get their consent for these experiments. Each subject was asked to drive the car for 60 minutes with a view to keep the car at the center of the cruising lane by maneuvering it with the steering wheel. Of the thirteen subjects, four struggled with mild drowsiness, while the remaining nine exhibited mild and deep drowsy episodes during the 1-hour driving session.

\subsection{Indirect measurement of alertness}

To investigate the relationship between the measured EEG signals and subject's cognitive state, and to quantify the level of the subject's alertness, in our previous studies $[19,20]$, we have defined an indirect index of the subject's alertness level (driving performance) as the deviation between the center of the vehicle and the center of the cruising lane. Typically, the drowsiness level fluctuates with cycle lengths longer than 4 minutes [22-25], and hence we smooth the indirect alertness level index using a causal 90-second moving window advancing at 2 -second steps. This helps us to eliminate variance with cycle lengths shorter than 1-2 minutes. We emphasize that this index is used only to validate our approach, and it is not as an input to develop the model for the alert state of the subject.

\section{THE PROPOSED UNSUPERVISED APPROACH}

It is recognised that the changes in EEG spectra in the thetaband $(4 \sim 7 \mathrm{~Hz})$ and alpha-band $(8 \sim 11 \mathrm{~Hz})$ reflect changes in the cognitive and memory performance [26]. Other studies have reported that EEG power spectra at the thetaband $[25,27]$ and/or alpha-band $[28,29]$ are associated with drowsiness, and EEG log power and subject's driving performance are largely linearly related. These findings have motivated us to derive the alert models of the driver using the alpha-band and theta-band EEG power spectrum computed using $\mathrm{OZ}$ channel output recorded in the first few minutes of driving. The choice of the $\mathrm{OZ}$ channel is explained in Section 4. We emphasize that the few minutes of data used to find the alert model are not necessarily collected from the very beginning of the driving session because different factors, for example, if the driver walks a few meters to reach the garage, may influence the EEG signal generated at the very beginning. The specific window to be used for generating the alert model is selected by Mardia test (explained later) [30]. We assume that if the subject/driver is in an alert state, then the EEG power spectra relating to theta-band (as well as that relating to alpha-band) would follow a multivariate normal distribution. The parameters of the multivariate normal distributions characterise the models. Using the alpha-band and theta-band EEG powers, we identify two normal-distribution-based models. Then, we assess the deviation of the current state of the subject from the alert model using Mahalanobis distance (MD). We assume that when the subject continues to remain alert, 
his/her EEG power should resemble the sample data used to generate the model and hence would match the alert model or template. If the subject becomes drowsy, then its power spectra in the alpha-band (and also in theta-band) will deviate from the respective model and hence MD will increase. With a view to reduce the effect of spurious noise, MDs are smoothed over a 90-second moving windows, the window is moved by 2 -second steps. We then study the relationship between smoothed Mahalanobis distance and subject's driving performance by computing the correlation between the two. Figure 2 shows the overall flow of the EEG data analysis. In Figure 2, note that, after the models are identified, the preprocessed alpha-band and theta-band power data directly go to the blocks for computation of MDA and MDT, respectively. MDT and MDA are measures of deviations of the subject's present state from the respective models, this will be clarified later. The block for computation of MDC makes a linear combination of MDT and MDA. Finally, the three, MDA, MDT, and MDC, are used in correlation analysis with the driver's performance. We now explain the various major tasks in the model development and the use of the model in the following sections.

\subsection{Smoothing of the power spectra}

We use a componentwise median filter for smoothing the power spectrum data. We compute one data vector (a vector with power spectrum) in 20 dimensions using 2-second signal and fast Fourier transform (FFT). Thus, we consider 500point Hanning windows without overlap. Each windowed 500 -point epoch is now subdivided into 16 subepochs each with 125 points using a Hanning window. Each subepoch is shifted by 25 points. For example, the first subepoch uses points 1 through 125, the second subepoch uses points 26 through 150, and so on. Each subepoch is then extended to 256 points by zero padding for a 256-point FFT. A moving median (computed using the 16 subepochs) filter is used to minimise the presence of artifacts in the EEG records of all subwindows. The median filter is realised by computing the median of each component. In other words, for 2-second signal, we have generated 16 vectors, each in 20 dimensions. Then, we generate a new vector in 20 dimensions, where the $i$ th component is the median value of the $i$ th component of the 16 vectors. We call this new vector the moving median filtered data. This process is repeated for every two seconds without overlap. The moving median filtered EEG power spectra are then converted to a logarithmic scale prior to further analysis. Logarithmic scaling linearizes the expected multiplicative effects of subcortical systems involved in wake-sleep regulation on EEG amplitudes [31]. Thus for each session, EEG log power time series at alpha-band as well as at theta-band with 2-second (500-point, an epoch) timeintervals are generated. These time series data are the inputs to our model.

\subsection{Computation of the alert model of the subject}

In our approach, for every subject in every driving session a new model will be constructed. Consequently, the variability between subjects as well as the intersession variability is no more important, because they are taken into account automatically. To develop the alert model we make a few mild but realistic assumptions as follows.

(1) The subject is usually very alert immediately after he/she starts the driving session.

(2) Subject's cognitive state can be characterised by the power spectrum of his/her EEG.

(3) When the person is in the alert state, he/she can be modeled reasonably well using a multivariate distribution of the power spectrum.

(4) The alert model expresses well the EEG spectra when the subject remains alert or returns to alert state from drowsiness.

One can argue that the subject may already be in a drowsy state when he/she begins driving. If that is really true, then that can be detected by checking the consistency between two alert models derived using data in two successive time intervals. In other words, we can check whether the two alert-models identification in two successive time intervals are statistically the same or not. If the subject was already in a drowsy state, then he/she will either move to a deep drowsy/sleepy state or will transit to an alert state. In both cases, the two models will not be statistically the same.

Here, we use a multivariate distribution to model the distribution of power spectrum in the alert state. In particular, every 2 seconds, we calculate the power spectrum vector in $p$ dimension (in our experiment $p=4$ (thetaband) or $p=5$ (alpha-band)). In this way, a set of $n=30$ data vectors $\left\{\mathbf{x}_{1}, \ldots, \mathbf{x}_{30}\right\}$ is generated in every minute. We use 3 minutes of spectral data to derive the alert model. The alert model is represented and characterised by a multivariate normal distribution $N\left(\mu, \Sigma^{2}\right)$, where $\mu$ is the mean vector and $\Sigma$ is the variance-covariance matrix.

We use the maximum likelihood estimates for $\mu$ and $\Sigma^{2}$. After finding the alert model, we check whether the EEG spectrum in the alpha-band (also in theta-band) indeed follows a multivariate normal using Mardia's test [31-33]. If the model passes the Mardia's test, we accept that model as the alert model. Otherwise, we move the data window by one minute and again use the next 3 minutes of data to derive and validate the model using Mardia's test. Once a model is built, a significant deviation from the model can be taken as a departure from alertness. Note that we are saying "departure from alertness" which is not necessarily drowsiness. For example, the subject could be excited over a continued conversation over a mobile phone. In this case, although the person is not drowsy, he/she is not alert as far as the driving task is concerned and hence needs to be cautioned. Thus our approach is more useful than typical drowsiness detection systems. A consistent and significant deviation for some time can be taken as an indicator of drowsiness.

For the sake of completeness, we briefly explain the Mardia's test of multivariate normality. Given a random 
TABLE 1: The average correlation between mahalanobis distance and driving error of all subjects for different channels.

\begin{tabular}{lcccccccccc}
\hline Pole & F7 & F3 & FZ & F4 & F8 & FT7 & FC3 & FCZ & FC4 & FT8 \\
\hline MDA & 0.52 & 0.45 & 0.59 & 0.47 & 0.47 & 0.53 & 0.59 & 0.60 & 0.56 & 0.48 \\
MDT & 0.13 & 0.25 & 0.42 & 0.23 & 0.09 & 0.27 & 0.56 & 0.60 & 0.46 & 0.13 \\
\hline Pole & T3 & C3 & CZ & C4 & T4 & TP7 & CP3 & CPZ & CP4 & TP8 \\
\hline MDA & 0.60 & 0.58 & 0.58 & 0.54 & 0.48 & 0.54 & 0.57 & 0.57 & 0.51 & 0.52 \\
MDT & 0.38 & 0.60 & 0.63 & 0.54 & 0.34 & 0.53 & 0.58 & 0.67 & 0.55 & 0.53 \\
\hline Pole & P7 & P3 & PZ & P4 & P8 & O1 & OZ & O2 & - & - \\
\hline MDA & 0.56 & 0.56 & 0.52 & 0.53 & 0.53 & 0.60 & 0.64 & 0.64 & - & - \\
MDT & 0.57 & 0.64 & 0.66 & 0.63 & 0.65 & 0.71 & 0.74 & 0.73 & - & - \\
\hline
\end{tabular}

sample, $X=\left\{\mathbf{x}_{1}, \ldots, \mathbf{x}_{n}\right\}$ in $R^{p}$, Mardia [32-34] defined the $p$-variate skewness and kurtosis as

$$
\begin{aligned}
& b_{1, p}=\frac{1}{n^{2}} \sum_{i=1}^{n} \sum_{j=1}^{n}\left\{\left(\mathbf{x}_{i}-\overline{\mathbf{x}}\right)^{\prime} S^{-1}\left(\mathbf{x}_{j}-\overline{\mathbf{x}}\right)\right\}^{3}, \\
& b_{2, p}=\frac{1}{n} \sum_{i=1}^{n}\left\{\left(\mathbf{x}_{i}-\overline{\mathbf{x}}\right)^{\prime} S^{-1}\left(\mathbf{x}_{i}-\overline{\mathbf{x}}\right)\right\}^{2} .
\end{aligned}
$$

In (1) $\overline{\mathbf{x}}$ and $S$ represent the sample mean vector and covariance matrix, respectively. In the case of univariate data, $b_{1, p}$ and $b_{2, p}$ reduce to the usual univariate measures skewness and kurtosis, respectively. If the sample is obtained from a multivariate normal distribution, then the limiting distribution of $b_{1, p}$ is a chi-square with $p(p+1)(p+$ $2) / 6$ degrees of freedom, while that of $\sqrt{n}\left(b_{2, p}-p(p+\right.$ 2)) $/ 8 \sqrt{p(p+2)}$ is $N(0,1)$. Hence, we can use these statistics to test multivariate normality. In all our experiments, we have used the routines available for Mardia's test in the Rpackage [35].

After the alert model is found, we use it to assess the subject's cognitive state. This is done by finding how the subject's present state, as represented by the EEG power spectra, is different from the state represented by the alert model. The deviation of the present state from the model is computed using Mahalanobis distance [36] that can account for the covariance between variables while computing the distance. Let the alert model computed using the alphaband be represented by $(\overline{\mathbf{x}}, S)_{A}$ and that by the theta-band be represented by $(\overline{\mathbf{x}}, S)_{T}$. Let $\mathbf{x}$ be a vector representing the power spectra in the alpha-band (or in the theta-band) of the EEG of the subject at some time instant, then the deviation of the present state from the model is

$$
\operatorname{MD}^{*}(\mathbf{x})=\sqrt{(\mathbf{x}-\overline{\mathbf{x}})^{T} S^{-1}(\mathbf{x}-\overline{\mathbf{x}})}
$$

In (2) if we use the alpha-band model, then $*$ is $A$, and for the theta-band model and data, $*$ will be $T$. Thus, the deviation from the alpha-band model will be denoted by MDA and that for the theta-band model will be denoted by MDT. Similar to the preprocessing of the indirect alertness level index (driving performance), the MDA/MDT is also smoothed by the moving average method using a window with 45 values (i.e., the average is over 90 seconds). The moving average window is shifted by just one value (i.e., 2 seconds). For a better visual display, we have scaled the $\mathrm{MD}^{*}$ values by subtracting the average $\mathrm{MD}^{*}$ computed over the training data used for finding the alert model.

We will see later that the deviation from either the alphaband model (i.e., MDA) or the theta-band model (i.e., MDT) can be used to detect departure from the alart cognitive state. This raises a natural question: can a combined use of MDA and MDT do a better job than individual ones. To explore such a possibility we use a linear combination MDA and MDT to compute a combined measure of deviation as $\mathrm{MDC}=a \cdot \mathrm{MDA}+(1-a) \cdot \mathrm{MDT}, 0 \leq a \leq 1$.

Now, in order to demonstrate that $\mathrm{MD}^{*}(*=\mathrm{MDA} /$ MDT/MDC) can be used to detect changes in the cognitive states, we compute the linear correlation between the alertness level index $(d)$ and the smoothed Mahalanobis distance $\left(\mathrm{MD}^{*}\right)$. In our subsequent discussion $\mathrm{MD}^{*}$ will represent the smoothed deviations, that is, the smoothed values of MDA, MDT, and MDC as the case may be. The correlation coefficient is defined as

$$
\operatorname{Corr}_{d, \mathrm{MD}^{*}}=\frac{\sum(d-\bar{d})\left(\mathrm{MD}^{*}-\overline{\mathrm{MD}^{*}}\right)}{\sqrt{\sum(d-\bar{d})^{2} \sqrt{\left(\mathrm{MD}^{*}-\overline{\mathrm{MD}^{*}}\right)^{2}}}}
$$

\section{EXPERIMENT RESULTS}

There are a few important issues to be resolved before we can proceed with the detailed analysis. The first issue is how to decide the optimal window size for feature extraction (computing FFT). For this, we have tried various choices and have found that 2-second signal does a reasonably good job and that is what we use here. Note that one can use a more systematic approach using training and validation data to find the optimal window size. The next issue is the choice of channels to be used for analysis. We have data from 28 EEG channels and we wanted to use only one channel. To find the most useful channel for the problem at hand, for each channel we compute the average correlation (averaged over all subjects) between MDA and the driving performance. Similarly, we also compute the average correlation between MDT and the driving performance. These correlation values are summarised in Table 1 . Table 1 reveals that the highest correlation occurs for channel OZ both with MDT and MDA. This suggests that channel OZ is better than other 


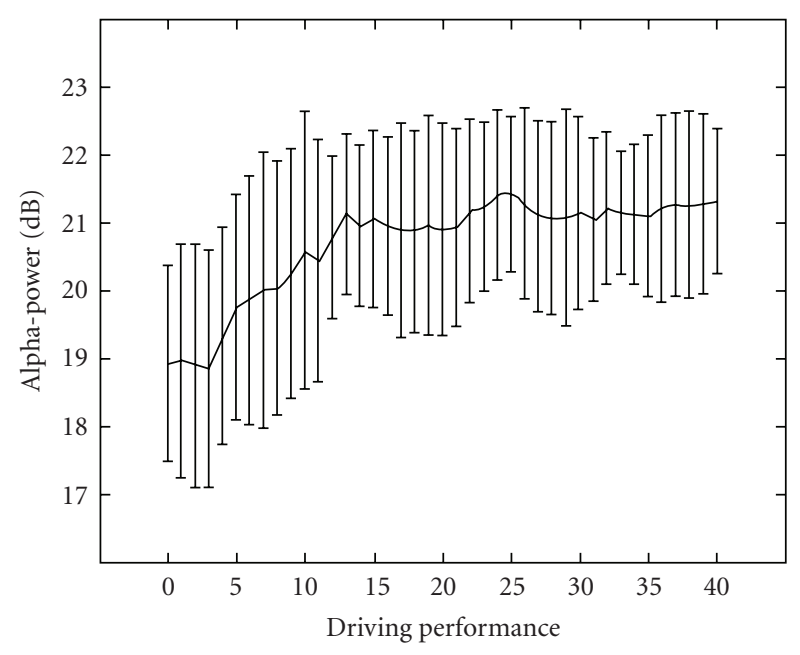

(a)

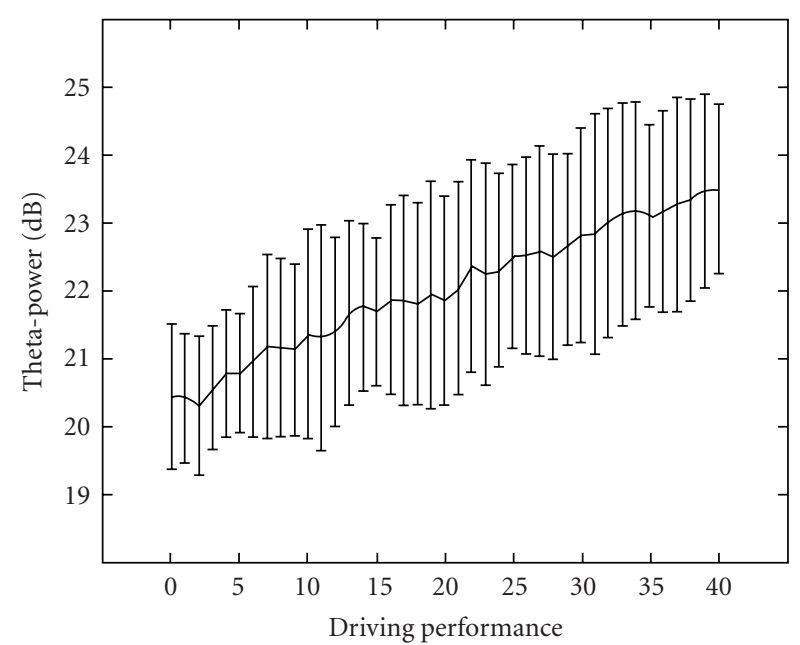

(b)

FIgURE 3: Error-sorted EEG spectra at OZ over 13 sessions. (a) The solid lines represent the grand mean power spectra and the dotted lines represent the standard deviations of the power spectra. When the driving error increases from 0 to 20 , the mean of alpha-power $(8 \sim 12 \mathrm{~Hz})$ rises sharply and monotonically from 19 to $21 \mathrm{~dB}$, after which it remains more or less stable near $2 \mathrm{~dB}$ above the baseline. (b) The mean of theta-power $(4 \sim 7 \mathrm{~Hz})$ increases monotonically and steadily from 20 to $23 \mathrm{~dB}$ as the driving error increases (alertness to deep drowsiness).

channels in discriminating departure from alertness. The channels $\mathrm{O} 1$ and $\mathrm{O} 2$ which are neighbors of $\mathrm{OZ}$ also exhibit a very high correlation. Since we have decided to use only one channel, we have chosen channel OZ for further study.

To investigate the relationship between the driver's performance and the concurrent changes in the EEG spectrum, we have sorted the EEG power spectra in alpha-band by smoothed driving error. The similar sorting is also done for power in the theta-band. Figure 3(a) depicts the relation between the alpha-power and the driving error, while Figure 3(b) displays the same for theta-power. Figure 3(a) reveals that when the driving error increases from 0 to 20 , the mean of alpha-power $(8 \sim 12 \mathrm{~Hz})$ rises sharply and monotonically from 19 to $21 \mathrm{~dB}$, after that it slowly goes down a little bit. While for the theta-power (Figure 3(b)), the mean power $(4 \sim 7 \mathrm{~Hz})$ increases monotonically and steadily from 20 to $23 \mathrm{~dB}$ as the driving error increases (alertness to deep drowsiness).

Our alert model does not use EEG power, but MDT and MDA. So, next we check how strongly MDA and MDT are correlated with the driving performance. Figure 4(a) shows the relation between driving error and MDA (across the 13 test subjects/sessions) while Figure 4(b) exhibits the same for MDT. It is interesting to see that Figures 3 and 4 exhibit almost the same behaviour; in fact, for Figure 4(b) we find that compared to Figure 3(b), the average MDT increases more steadily with driving performance.

Can we say that the use of $\mathrm{MD}^{*}$ would be more useful than the use of alpha- and theta-power? To address this question, for every subject we have computed the correlation between power (in alpha- and theta-bands) and driving error and also the correlation between $\mathrm{MD}^{*}$ (MDA and MDT) and driving error. Table 2 summarises the correlation values. Table 2 reveals that of the 26 sets of correlation values, in
TABLE 2: The comparison of the correlation between power and driving performance and $\mathrm{MD}^{*}$ and driving performance for channel OZ.

\begin{tabular}{lcc}
\hline Subjects & $\begin{array}{c}\text { Power correlation } \\
\text { (alpha/theta) }\end{array}$ & $\begin{array}{c}\text { Distance correlation } \\
\text { (MDA/MDT) }\end{array}$ \\
\hline S1 & $0.57 / 0.34$ & $0.75 / 0.73$ \\
S2 & $0.70 / 0.51$ & $0.69 / 0.47$ \\
S3 & $0.63 / 0.60$ & $0.67 / 0.65$ \\
S4 & $0.26 / 0.14$ & $0.47 / 0.41$ \\
S5 & $0.66 / 0.79$ & $0.62 / 0.85$ \\
S6 & $0.26 / 0.88$ & $0.63 / 0.85$ \\
S7 & $0.66 / 0.97$ & $0.57 / 0.96$ \\
S8 & $0.04 / 0.72$ & $0.76 / 0.80$ \\
S9 & $0.41 / 0.78$ & $0.39 / 0.77$ \\
S10 & $0.60 / 0.87$ & $0.76 / 0.88$ \\
S11 & $0.40 / 0.57$ & $0.53 / 0.90$ \\
S12 & $0.35 / 0.52$ & $0.24 / 0.62$ \\
S13 & $0.40 / 0.94$ & $0.45 / 0.95$ \\
\hline Average & $0.45 / 0.62$ & $0.58 / 0.76$ \\
\hline
\end{tabular}

16 cases the correlation has increased with MD*. In a few cases, the increase in correlation is very high. For example, with subject S8, the correlation with alpha-power is only 0.04 while that with MDA is 0.76 . Similarly, for S6, the alpha-power correlation is 0.26 which enhances to 0.63 for MDA. This clearly indicates the effectiveness of the alert model. Table 2 also displays the average correlation values. The average correlation with deviations from the model is increased by about $30 \%$ for alpha-band, while that for the theta-band is increased by about $23 \%$. 


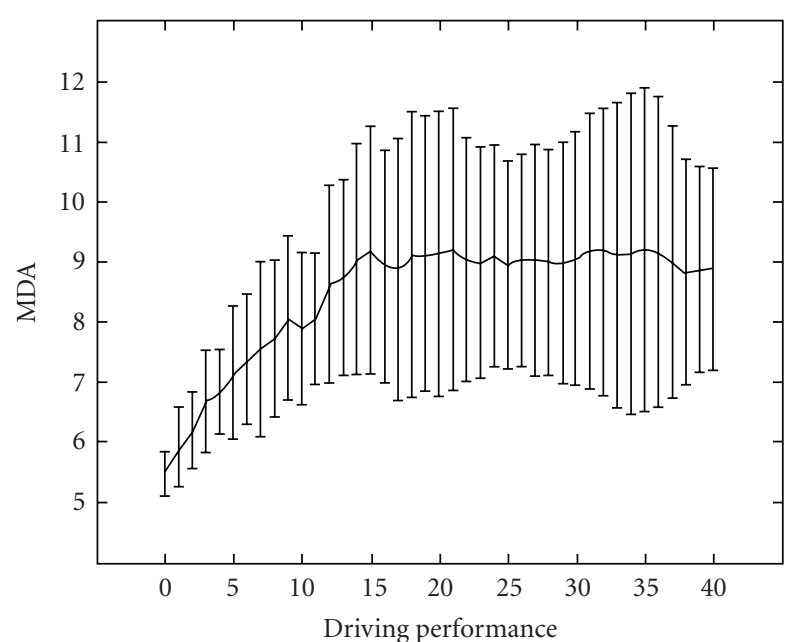

(a)

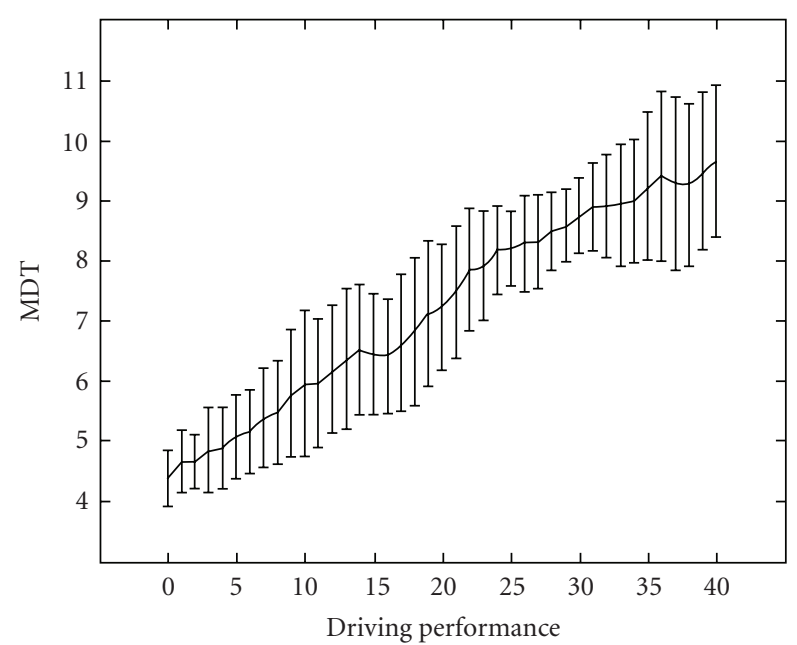

(b)

FIGURE 4: Error-sorted MD for different sessions. (a) The solid lines represent the grand mean MD and the dotted lines represent its standard deviations. When the driving error increases from 0 to 20, the MDA rises sharply and monotonically from 5 to 9 , after which it remains more or less stable. (b) The MDT increases monotonically and steadily from 4 to 9 as the driving error increases (alertness to deep drowsiness).

TABLE 3: All combination correlations of all subjects by using OZ channel

\begin{tabular}{|c|c|c|c|c|c|c|}
\hline Subjects & $\begin{array}{c}\text { Correlation } \\
\text { (MDA/MDT) }\end{array}$ & $\begin{array}{l}\text { Correlation } 0.1^{*} \\
\text { MDA } 0.9^{*} \mathrm{MDT}\end{array}$ & $\begin{array}{l}\text { Correlation } 0.3^{*} \\
\text { MDA } 0.7^{*} \text { MDT }\end{array}$ & $\begin{array}{l}\text { Correlation } 0.5^{*} \\
\text { MDA } 0.5^{*} \text { MDT }\end{array}$ & $\begin{array}{l}\text { Correlation } 0.7^{*} \\
\text { MDA } 0.3^{*} \text { MDT }\end{array}$ & $\begin{array}{l}\text { Correlation } 0.9^{*} \\
\text { MDA } 0.1^{*} \mathrm{MDT}\end{array}$ \\
\hline S1 & $0.75 / 0.73$ & 0.75 & 0.78 & 0.77 & 0.77 & 0.75 \\
\hline S2 & $0.69 / 0.47$ & 0.59 & 0.67 & 0.69 & 0.69 & 0.69 \\
\hline S3 & $0.67 / 0.65$ & 0.66 & 0.68 & 0.69 & 0.69 & 0.68 \\
\hline S4 & $0.47 / 0.41$ & 0.43 & 0.46 & 0.48 & 0.48 & 0.47 \\
\hline S5 & $0.62 / 0.85$ & 0.84 & 0.80 & 0.75 & 0.70 & 0.65 \\
\hline S6 & $0.63 / 0.85$ & 0.86 & 0.86 & 0.85 & 0.83 & 0.74 \\
\hline S7 & $0.57 / 0.96$ & 0.95 & 0.92 & 0.84 & 0.74 & 0.62 \\
\hline S8 & $0.76 / 0.80$ & 0.80 & 0.81 & 0.82 & 0.81 & 0.79 \\
\hline S9 & $0.39 / 0.77$ & 0.77 & 0.75 & 0.69 & 0.58 & 0.45 \\
\hline S10 & $0.76 / 0.88$ & 0.88 & 0.88 & 0.87 & 0.85 & 0.81 \\
\hline S11 & $0.53 / 0.90$ & 0.92 & 0.90 & 0.86 & 0.74 & 0.60 \\
\hline S12 & $0.24 / 0.62$ & 0.64 & 0.65 & 0.67 & 0.65 & 0.45 \\
\hline $\mathrm{S} 13$ & $0.45 / 0.95$ & 0.95 & 0.93 & 0.87 & 0.75 & 0.56 \\
\hline Average & $0.58 / 0.76$ & 0.77 & 0.78 & 0.76 & 0.71 & 0.64 \\
\hline
\end{tabular}

\subsection{Linear combination of model deviations}

The analysis above provides strong and convincing evidence that changes in the driving performance during a long driving session are related to the changes in the EEG power in the alpha- and theta-bands. In the given experimental setup, higher driving error corresponds to departure from alert state of mind. Thus, departures from alert cognitive state are reflected in the EEG power of the alpha- and thetabands. The change (correlation) is more strongly visible in the deviations from the alert model derived based on multivariate normal distribution. We have experimented with two models, one based on alpha-band and other based on the theta-band. Both appear quite effective. But can we improve it further using the two bands/models together? Figure 5 displays the MDT and MDA as a function of driving error. From these figures as well as from Table 2, we find that driving errors of mild drowsy cases are more strongly related to MDA, while MDT is highly correlated with driving performance for cases when the subject went to a deep drowsy state. Thus, if we can use the right model based on alpha-band or theta-band, we can do a better detection. But in reality, we will not know beforehand which model to use. So a combined model could be more useful.

To examine this possibility, we consider a very simple liner combination of MDA and MDT as MDC $=a \cdot \mathrm{MDA}+$ $(1-a) \cdot \mathrm{MDT}, 0 \leq a \leq 1$. There are infinitely possible choices for the constant $a$ in the linear combination. We have 

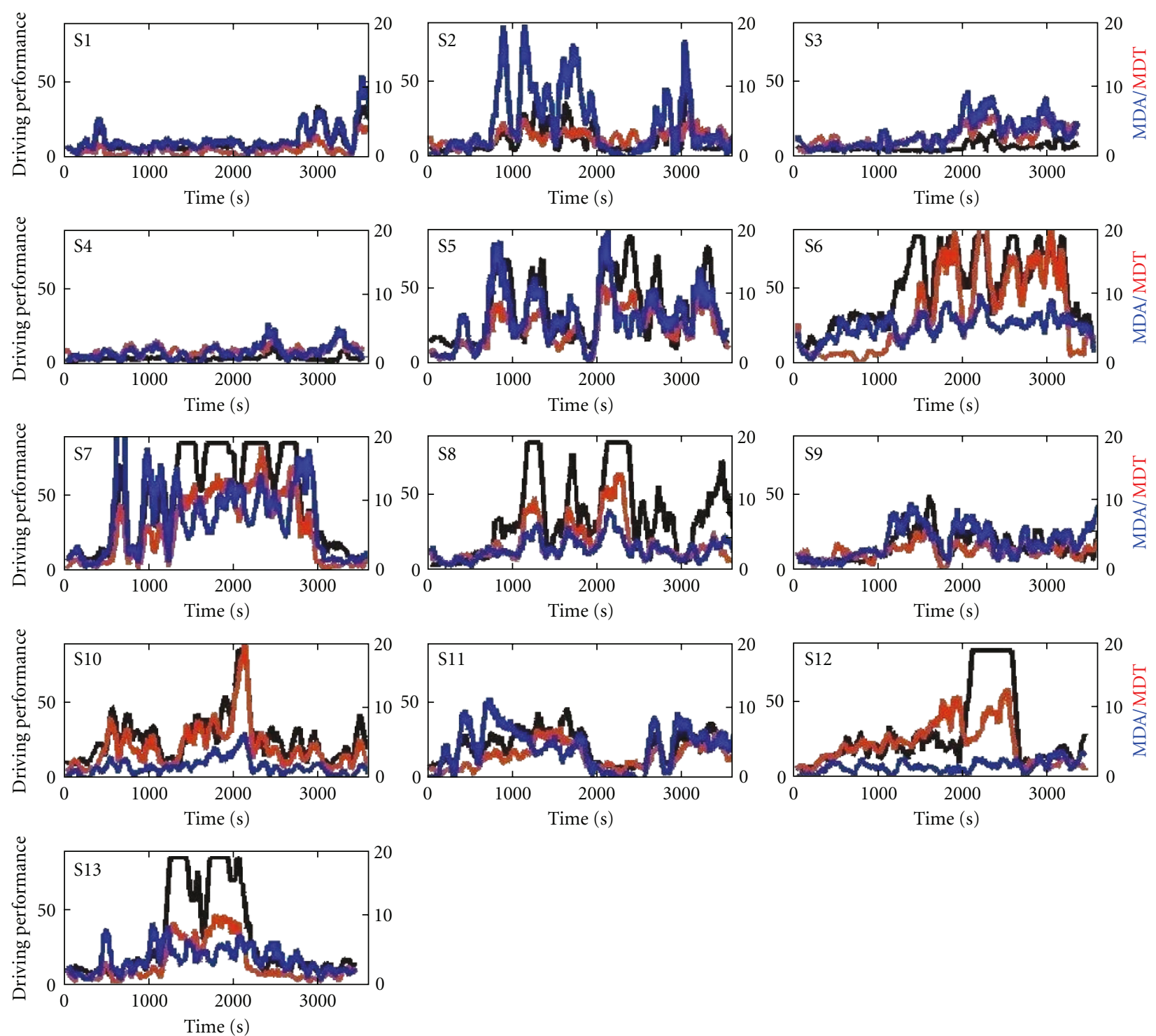

Driving performance
MDA
MDT

FIGURE 5: MDT and MDA versus actual driving performance. Some subjects such as S1 experienced mild drowsiness and MDA is strongly correlated with subject's driving performance. Another subject, S10, was in a deep drowsy state and for this subject MDT is highly correlated with its driving performance.

used a grid search starting from $a=0$ to $a=1$ with an increment of 0.1 and for every such linear combination we have computed the correlation of MDC with a driving error. Based on the limited dataset that we have used, we found $a=0.3$ as the best choice. Table 3 lists the correlation values for a few illustrative cases. Note that in the second column we have two correlation values $x / y$, where $x$ corresponds to MDA (i.e., $a=1$ ) and $y$ corresponds to MDT (i.e., $a=0$ ). The bottom row in Table 3 shows the average correlations of all combinations from thirteen subjects. Displays the average correlation values. Although the improvement in average correlation is marginal, what is important is that for the combined model for both deep drowsy and mild drowsy cases we get a very good correlation. As anexample, for subject S9, if we use MDA, the correlation is only 0.39 , while using MDC, for all combinations the correlation is higher than that with MDA. This justifies the utility of the combined model. Figure 6 depicts the driving error and the MDC for all 13 subjects. It is clear from these figures that, on average, $\mathrm{MDC}$ is in more agreement with the driving error. 

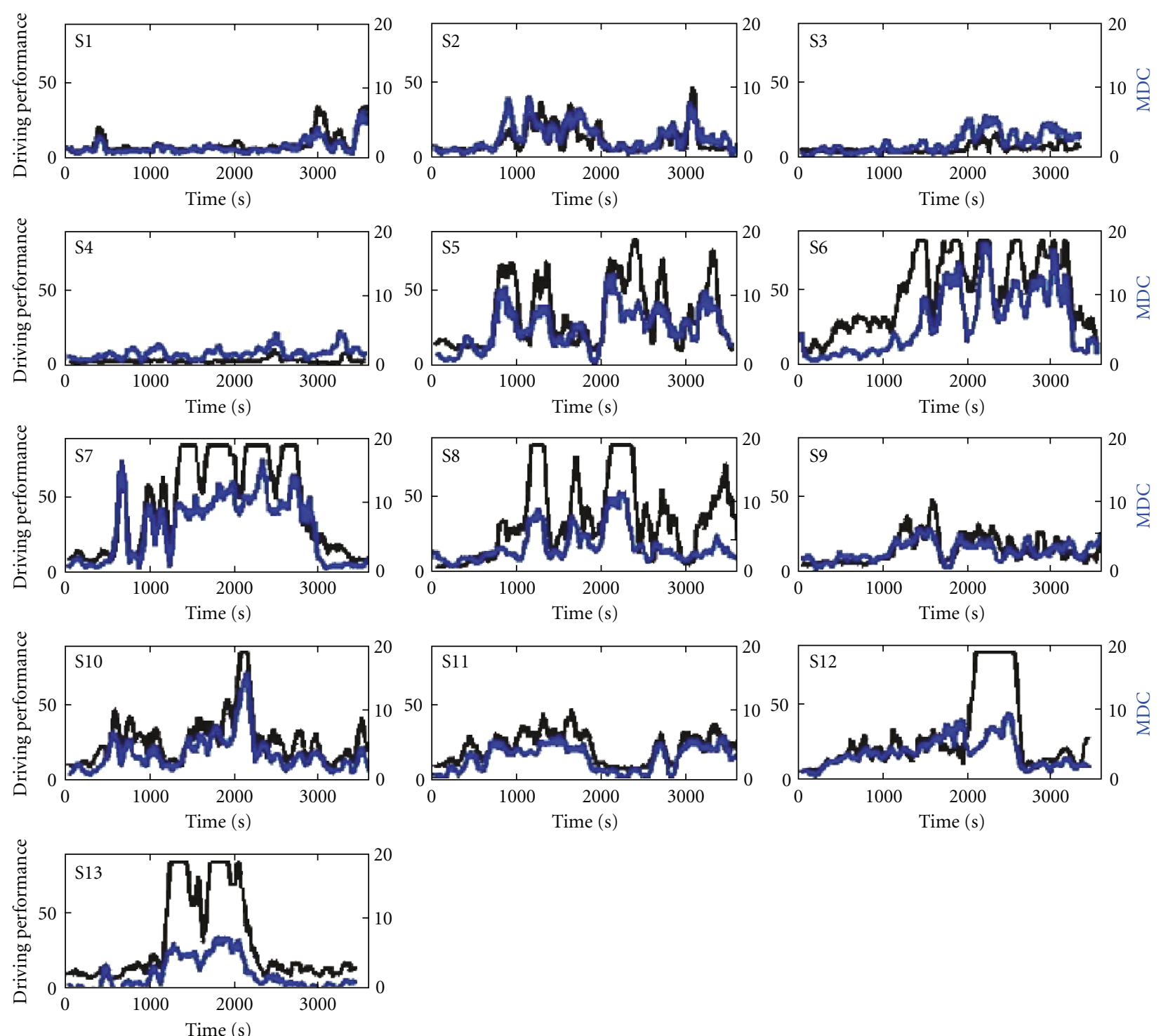

Driving performance

MDC

FIgure 6: The time series of MDC (0.3 MDA + 0.7 MDT) from OZ channel and the driving performance of all subjects. The black line represents driving performance and the blue line corresponds to MDC. The MDC is found to be highly correlated with driving performance.

\section{DISCUSSIONS}

We have assumed that when a subject starts driving, he is in an alert state. However, this may not necessarily be true. If the person is not in an alert state (i.e., he/she is in a drowsy state), then either he will move to a deep drowsy state or will get to the alert state with time. Thus his/her EEG power spectrum will change with time. This type of situations can be detected using a consistency check as explained earlier. For example, we can find two alert models of the person at time instant $t$ second and at $t+\delta$ second, where $\delta$ can be 180 seconds. If the person is in an alert state, then these two models will be statistically the same. So we can use such hypothesis testing to authenticate whether the person is in an alert state at the beginning or not. If desired, this can be further strengthened having a stored alert model. If the consistency check explained above fails, then we can check the similarity between the stored model and the model just found. If these two models are also significantly different, this will further suggest that the person is not in an alert state.

The dataset used in this study is not very big. From the 13 subjects, four subjects were mild drowsy during the driving experiments, while the remaining subjects went through episodes of mild drowsy to deep drowsy states. To demonstrate the effectiveness of this method, further investigation using a bigger set needs to be done.

We have used only OZ channel. Use of O1 and $\mathrm{O} 2$ along with $\mathrm{OZ}$ might improve the system performance. 
In this investigation, we have demonstrated the feasibility of an unsupervised subject and session independent approach to detect departure from alertness in driver. In future, we plan to identify thresholds on MDA/MDT/MDC which can be used to label the driver's cognitive state as alert/mild drowsy/deep drowsy. This will require some validation data as well as authentication by experts. Once this is done, such thresholds can be used in conjunction with the unsupervised method. We keep all these for our future investigation.

\section{CONCLUSIONS}

In this study, we propose an unsupervised approach that in every driving session generates a statistical model of the alert state of the subject using a very limited data obtained at the beginning of the driving session. Our model makes a few very realistic assumptions to derive the alert-state model. We assume that the EEG power spectrum in an alert state can be reasonably modeled using a multivariate normal distribution. The model is first validated statistically and then used to asses the cognitive state of the driver. A significant deviation from the model is taken as a departure from the alert state. We also attempt to find good choices of channel(s) and EEG features for assessing the drowsinessrelated EEG dynamics. We have found that OZ is an effective channel and the power spectra in the theta-band and alphaband have good discriminating power. We have derived three models: one based on alpha-band spectrum, one based on the theta-band power spectrum, and the third one combines the deviations of the subjects' present cognitive state from the two models. We have demonstrated that the deviation of the subjects present cognitive state from the alert model covaries with the driving performance which is an indirect measure of operators' changing levels of alertness when they perform a realistic driving task in a VR-based driving simulator. Unlike most supervised methods, our method can account for large individual and cross-session variability in EEG dynamics.

\section{REFERENCES}

[1] R. S. Huang, C. J. Kuo, L.-L. Tsai, and O. T. C. Chen, "EEG pattern recognition-arousal states detection and classification," in Proceedings of IEEE International Conference on Neural Networks (ICNN '96), vol. 2, pp. 641-646, Washington, DC, USA, June 1996.

[2] A. Vuckovic, V. Radivojevic, A. C. N. Chen, and D. Popovic, "Automatic recognition of alertness and drowsiness from EEG by an artificial neural network," Medical Engineering and Physics, vol. 24, no. 5, pp. 349-360, 2002.

[3] S. Roberts, I. Rezek, R. Everson, H. Stone, S. Wilson, and C. Aiford, "Automated assessment of vigilance using committees of radial basis function analysers," IEE Proceedings: Science, Measurement and Technology, vol. 147, no. 6, pp. 333-338, 2000.

[4] K. B. Khalifa, M. H. Bedoui, R. Raytchev, and M. Dogui, "A portable device for alertness detection," in Proceedings of the 1st Annual International Conference on Microtechnologies in Medicine and Biology (MMB '00), pp. 584-586, Lyon, France, October 2000.
[5] B. J. Wilson and T. D. Bracewell, "Alertness monitor using neural networks for EEG analysis," in Proceedings of the 10th IEEE Workshop on Neural Network for Signal Processing (NNSP '00), vol. 2, pp. 814-820, Sydney, Australia, December 2000.

[6] K. F. Van Orden, W. Limbert, S. Makeig, and T. P. Jung, "Eye activity correlates of workload during a visuospatial memory task," Human Factors, vol. 43, no. 1, pp. 111-121, 2001.

[7] M. Matousek and I. Petersen, "A method for assessing alertness fluctuations from EEG spectra," Electroencephalography and Clinical Neurophysiology, vol. 55, no. 1, pp. 108-113, 1983.

[8] J. Beatty, A. Greenberg, W. P. Deibler, and J. O'Hanlon, “Operant control of occipital theta rhythm affects performance, in a radar monitoring task," Science, vol. 183, no. 4127, pp. 871873, 1974.

[9] J. A. Stern, D. Boyer, and D. Schroeder, "Blink rate: a possible measure of fatigue," Human Factors, vol. 36, no. 2, pp. 285297, 1994.

[10] D. Schmidt, L. A. Abel, L. F. Dell'Osso, and R. B. Daroff, "Saccadic velocity characteristics: intrinsic variability and fatigue," Aviation Space and Environmental Medicine, vol. 50, no. 4, pp. 393-395, 1979.

[11] D. K. McGregor and J. A. Stern, "Time on task and blink effects on saccade duration,” Ergonomics, vol. 39, no. 4, pp. 649-660, 1996.

[12] K. F. Van Orden, T.-P. Jung, and S. Makeig, "Combined eye activity measures accurately estimate changes in sustained visual task performance," Biological Psychology, vol. 52, no. 3, pp. 221-240, 2000.

[13] C. Jutten and J. Herault, "Blind separation of sources-part I: an adaptive algorithm based on neuromimetic architecture," Signal Processing, vol. 24, no. 1, pp. 1-10, 1991.

[14] P. Comon, "Independent component analysis: a new concept?” Signal Processing, vol. 36, no. 3, pp. 287-314, 1994.

[15] A. J. Bell and T. J. Sejnowski, "An information-maximization approach to blind separation and blind deconvolution," Neural Computation, vol. 7, no. 6, pp. 1129-1159, 1995.

[16] M. Girolami, "An alternative perspective on adaptive independent component analysis algorithms," Neural Computation, vol. 10, no. 8, pp. 2103-2114, 1998.

[17] T.-W. Lee, M. Girolami, and T. J. Sejnowski, "Independent component analysis using an extended infomax algorithm for mixed sub-Gaussian and super-Gaussian sources," Neural Computation, vol. 11, no. 2, pp. 417-441, 1999.

[18] C. T. Lin, N. R. Pal, C. Y. Chuang, T. P. Jung, L. W. Ko, and S. F. Liang, "An EEG-based subject- and session-independent drowsiness detection," in Proceedings of International Joint Conference on Neural Networks (IJCNN) and the World Congress on Computational Intelligence (WCCI), pp. 34483454, Hong Kong, June 2008.

[19] C.-T. Lin, R.-C. Wu, T.-P. Jung, S.-F. Liang, and T.-Y. Huang, "Estimating driving performance based on EEG spectrum analysis," EURASIP Journal on Applied Signal Processing, vol. 2005, no. 19, pp. 3165-3174, 2005.

[20] C.-T. Lin, R.-C. Wu, S.-F. Liang, W.-H. Chao, Y.-J. Chen, and T.-P. Jung, "EEG-based drowsiness estimation for safety driving using independent component analysis," IEEE Transactions on Circuits and Systems I, vol. 52, no. 12, pp. 2726-2738, 2005.

[21] H. Ueno, M. Kaneda, and M. Tsukino, "Development of drowsiness detection system," in Proceedings of the Vehicle Navigation and Information Systems Conference (VNIS '94), pp. 15-20, Yokohama, Japan, August 1994. 
[22] S. Makeig and T.-P. Jung, "Changes in alertness are a principal component of variance in the EEG spectrum," NeuroReport, vol. 7, no. 1, pp. 213-216, 1995.

[23] T.-P. Jung, S. Makeig, M. Stensmo, and T. J. Sejnowski, "Estimating alertness from the EEG power spectrum," IEEE Transactions on Biomedical Engineering, vol. 44, no. 1, pp. 6069, 1997.

[24] S. Makeig and M. Inlow, "Lapses in alertness: coherence of fluctuations in performance and EEg spectrum," Electroencephalography and Clinical Neurophysiology, vol. 86, no. 1, pp. 23-35, 1993.

[25] S. Makeig and T.-P. Jung, "Tonic, phasic, and transient EEG correlates of auditory awareness in drowsiness," Cognitive Brain Research, vol. 4, no. 1, pp. 15-25, 1996.

[26] W. Klimesch, "EEG alpha and theta oscillations reflect cognitive and memory performance: a review and analysis," Brain Research Reviews, vol. 29, no. 2-3, pp. 169-195, 1999.

[27] S. Makeig, T.-P. Jung, and T. J. Sejnowski, "Awareness during drowsiness: dynamics and electrophysiological correlates," Canadian Journal of Experimental Psychology, vol. 54, no. 4, pp. 266-273, 2000.

[28] M. A. Schier, "Changes in EEG alpha power during simulated driving: a demonstration," International Journal of Psychophysiology, vol. 37, no. 2, pp. 155-162, 2000.

[29] S.-L. Joutsiniemi, S. Kaski, and T. A. Larsen, "Self-organizing map in recognition of topographic patterns of EEG spectra," IEEE Transactions on Biomedical Engineering, vol. 42, no. 11, pp. 1062-1068, 1995.

[30] K. V. Mardia, "Mardia's test of multinormality," in Encyclopedia of Statistical Sciences, S. Kotz and N. L. Johnson, Eds., vol. 5, pp. 217-221, John Wiley \& Sons, New York, NY, USA, 1985.

[31] M. Steriade, "Central core modulation of spontaneous oscillations and sensory transmission in thalamocortical systems," Current Opinion in Neurobiology, vol. 3, no. 4, pp. 619-625, 1993.

[32] K. V. Mardia, "Measures of multivariate skewness and kurtosis with applications," Biometrika, vol. 57, no. 3, pp. 519-530, 1970.

[33] K. V. Mardia, "Applications of some measures of multivariate skewness and kurtosis in testing normality and robustness studies," Sankhya B, vol. 36, no. 2, pp. 115-128, 1974.

[34] K. V. Mardia, "Tests of univariate and multivariate normality," in Handbook of Statistics, S. Kotz, Ed., vol. 1, pp. 279-320, North-Holland, Amsterdam, The Netherlands, 1980.

[35] http://www.r-project.org/.

[36] P. C. Mahalanobis, "Generalized distance in statistics," Proceedings of the National Institute of Science of India, pp. 49-55, 1936. 